\section{How to review a paper for Heart}

\author{
Catherine M Otto
}

Peer review is a core component of the scientific method. Pre-publication peer review for a medical journal serves two purposes. First, the review process assists the editorial team in selecting which papers should be published, based on several factors such as originality, scientific validity, match with the journal's scope and readers' interest. Second, peer review improves the quality of the final published papers, particularly in terms of presentation of the study design, data analysis and research results. Some aspects of the review process are similar across scientific journals, while others are specific to the focus of each journal. ${ }^{1-4}$ This article presents suggestions for reviewers for Heart, an international journal that publishes clinical and translational cardiovascular research.

Most of us have experienced the peerreview process both as an author and as a reviewer. Authors of previous publications are the peer reviewers for submitted manuscripts; your peer reviewers in the future will be the authors of papers you review today. We should be courteous to each other and respectful of scientific work. Part of that respect is an impartial and fair review. Write your review in a style as if you have your name declared, as many journals now request. ${ }^{3}$ Authors appreciate a short review cycle; currently, the rate-limiting step in the review cycle is the time that reviewers take to respond and submit their reviews. Reading a paper and writing the review typically only takes 2-3 hours; if you have time and the paper is within your area of expertise, accept right away and download the file to read as soon as possible. Submit your review on time, or even early. If you are unable to review, decline immediately so the editors can invite an alternate reviewer.

My approach to reviewing a paper is to first read the abstract. Many readers only look at abstracts; therefore, it needs to present the hypothesis, research design and results in a succinct, accurate and clear format. Next, I simply read the paper, looking carefully at the tables and figures, without taking notes or judging the research at this point. Then, as I think about the paper, key considerations are

Correspondence to Professor Catherine M Otto, Division of Cardiology, University of Washington, Seattle, WA 98195, USA; cmotto@u.washington.edu the originality and importance of the research hypothesis, a clearly presented and appropriate study design, convincing and credible data that add to our current knowledge, and the likelihood that these data will ultimately lead to improved patient care and clinical outcomes. If needed, a quick look online helps in putting the current study into context, particularly in terms of originality. After thinking about these primary issues, it is time to go back to the paper and take notes, section by section, based on the criteria indicated below.

The purpose of the introduction is to concisely present the background that prompted this research, highlighting areas of uncertainty in our current knowledge and then to provide a well-articulated hypothesis that is testable with this patient population and study design. The study should address an important clinical or scientific question. In addition, the study should be of interest to readers of Heart and fall within the scope of this journal. Obviously, authors submit only research related to cardiovascular disease to Heart. However, within this broad category, Heart is most interested in topics of interest to practicing clinicians, including patient-based research, translational studies and more basic studies likely to soon impact clinical care. On the other hand, articles that address technical aspects of a specific diagnostic or therapeutic approach might be better suited to a journal focusing on that cardiology sub-specialty.

The methods section allows reviewers and readers to evaluate the quality and relevance of the study results. Enough detail is needed to allow replication of the study results by future investigators with the order and content of the methods section exactly paralleling the results section. Basic components of a clinical cardiovascular research study include descriptions of the size and selection criteria for the study population; details of data acquisition, such as laboratory testing, genetic analysis or imaging measurements; definitions and methods for ascertaining clinical end points; and the statistical approach used for data analysis. Research involving human subjects must always provide verification of appropriate informed consent and institutional review board approval.
Clinical investigation is complex and the specific methodology depends on the focus of the research investigation. Heart recommends the use of research checklists, such as those developed by the Equator network, which provide established standards for reporting different types of research. ${ }^{5}$ Reviewers should look at the statistical methods used in the study to ensure that the relevant clinical parameters and end points are included in the analysis. Because most reviewers are not expert in statistical analysis, many journals, including Heart, also ask for a separate formal statistical review. The comments and suggestions of the statistical reviewer often substantially improve the presentation of the data and increase the confidence that the study findings are valid. In addition, a statistical review may reveal potential limitations or weaknesses that can be acknowledged in the paper to ensure that future studies focus on these areas of uncertainty.

Presenting the study results within the space constraints of a journal article can be challenging, yet a short presentation will increase the study's impact because more readers and researchers will be able to grasp the key findings and their implications. Detailed tables are the primary approach for presenting numerical research data. Well-designed tables provide information about the range and data distribution along with mean or median values both in the entire study group and in pre-defined subgroups. There should be no overlap in information between the text, tables and figures. When additional space is needed, most journals, including Heart, encourage online supplementary material.

Figures and graphs can provide more sophisticated data displays than shown by numbers in the text or tables. For example, standard life table graphs show survival (or event) rates over time for each group of research subjects. Forest plots provide an intuitive and quantitative display of relative risks, showing multiple parameters from a single study or comparing effects across studies. Graphical displays should seek to maximise the data included, for example, with numbers of subjects at risk at each time point on life table graphs and confidence intervals for odds ratios on forest plots. Graphical displays, such as bar graphs, that show no more data than can be provided by a simple listing of numbers are discouraged. It is preferable to use graphs that show the distribution of the data, such as box-and-whiskers plot, whenever possible. Reviewers should look carefully at tables 
and figures to ensure that all data elements are presented and that figures show the maximum amount of data in an unambiguous and intuitive format.

Reading the discussion is often the easiest part of reviewer's job. Good discussions briefly summarise the key study findings, put these findings in context of previous publications and current practice, point out limitations in the current study and comment on potential clinical implications. Precision in wording is important; a common mistake is for authors to use words that imply causation, rather than a simple association between a clinical variable and an outcome. For example, appropriate wording for data from a randomised trial is that treatment 'reduced' the clinical end point, implying a cause-and-effect relationship between the treatment and the end point. In contrast, for an observational study, the wording only should indicate that the treatment 'was associated with a lower rate' of the end point. Reviewers should ensure that the author's conclusions are justified by the data presented and that wide-sweeping clinical recommendations are avoided. Typically, controversial issues and future directions are better addressed in an accompanying editorial; Heart often invites one of the reviewers to write that editorial.

Final considerations for the reviewer are to check briefly that no key references have been omitted. Then look again at the abstract to ensure it accurately and completely reflects the data in manuscript and that numerical absolute, as well as relative, risks are provided whenever possible. Check that the abstract conclusion is balanced and matches the conclusions in the paper itself.

When submitting the review, organise your thoughts based on your review notes. It often is easier to write the comments to the authors first. Start with a very brief (one to two sentences) summary of the key points of the paper. Next, comment politely of whether the data are convincing and the conclusions appropriate. Then list ther concerns and suggestions for improvement, either grouped as major and minor comments or in a numbered list. There is no need to list every spelling, grammar or syntax error; journals have copy editors who will correct these issues before publication. Instead, focus on substantive issues and provide concrete suggestions to the authors on how to improve the presentation of their study. If you disagree with or consider a statement in the manuscript factually incorrect, a supporting reference should be provided.

The comments to the editors should not repeat your comments to the authors (the editors can see both of course) and can be short. The editors value your assessment of research originality, importance to readers of this journal, scientific reliability and potential clinical impact. Editors also appreciate a brief explanation of why you recommend acceptance or rejection, a frank appraisal of any major flaws, any revisions you consider essential if the paper is reconsidered and whether an editorial is needed to put this study in perspective. All major concerns raised with the editor should be adequately reflected in the comments to authors. If you have any potential conflicts of interest related to this review, those should be stated. Also, we all should remember that submitted articles are confidential until published. $^{6}$

If this all seems like a lot of work, stop and think about why we have peer review and what you expect from reviewers of your own papers. Compared to the amount of time and resources that we all put into medical research, it is a small and crucially important contribution to science to provide peer review of research within your area of expertise. In addition, being asked to review an article is a professional accomplishment, reflecting your recognition as an expert on that topic. Reviewers have insight into cutting-edge science and learn critical thinking, skills that enhance their own research and publications. Reviewers are part of the editorial team; those who do frequent high-quality reviews are often invited to join the editorial board and, perhaps, may become journal editors themselves one day.

Acknowledgements My sincere thanks to Paulus Kirchhof, David Newby, Antonio Luiz Pinho Ribeiro and David Shavelle for their helpful reviews of this editorial.

Competing interests None.

Provenance and peer review Commissioned; internally peer reviewed.

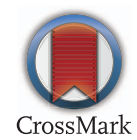

To cite Otto CM. Heart 2015;101:3-4.

Heart 2015;101:3-4.

doi:10.1136/heartjnl-2014-307135

\section{REFERENCES}

1 Bornmann L. Scientific peer review. Annu Rev Info Sci Technol 2011;45:197-245.

2 Demaria AN. Peer review: the weakest link. J Am Coll Cardiol 2010;55:1161-2.

3 Groves T, Loder E. Prepublication histories and open peer review at The BMJ. BMJ 2014;349:g5394.

4 Stahel PF, Moore EE. Peer review for biomedical publications: we can improve the system. BMC Med 2014;12:179.

5 Equator Network: Enhancing the QUALity and Transparency Of health Research. http://www. equator-network.org/ (accessed 21 Oct 2014).

6 Hames I; on behalf of the COPE Council. March 2013, V.1. COPE Ethical Guidelines for Peer Reviewers. http://publicationethics.org/files/Ethical_guidelines_for_ peer_reviewers_0.pdf (accessed 21 Oct 2014). 